\title{
PREVALÊNCIA DE LEISHMANIOSE CANINA EM CÃES DE ABRIGO NO MUNICIPIO DE UBERLÃNDIA - MINAS GERAIS - ESTUDO DE CASO
}

Fernanda Guimarães Rodrigues da Costa Reis ${ }^{1}$, Eduardo Francisco Ferreira de Andrade$^{1}$, Patrícia Alves Teixeira ${ }^{2}$, Danilo Guedes Junqueira Júnior ${ }^{3}$

${ }^{1}$ Bacharel em Medicina Veterinária do Centro Universitário do Triângulo, UberlândiaMG, Brasil.

2 Professora Mestre do curso de Medicina Veterinária do Centro Universitário do Triângulo, Uberlândia-MG, Brasil.

3 Professor Doutor do curso de Medicina Veterinária do Centro Universitário do Triângulo, Uberlândia-MG, Brasil.E-mail: dan_hp2002@yahoo.com.br

Recebido em: 15/02/2020 - Aprovado em: 15/03/2020 - Publicado em: 30/03/2020 DOI: 10.18677/EnciBio_2020A17

\begin{abstract}
RESUMO
A leishmaniose é um grande problema atualmente na saúde pública global e é uma doença endêmica em várias regiões do Brasil e do mundo, causada por protozoários do gênero Leishmania, sendo que as principais espécies que causam a leishmaniose visceral em cães são a Leishmania infantum e L. chagasi, que são disseminadas pela picada do mosquito flebotomíneo. Os maiores reservatórios da leishmaniose visceral são os cães domésticos. O aumento do número de cães abandonados em abrigo tem aumentado bastante, e o risco de disseminação de doenças entre esses animais em abrigo tornou-se constante. O presente estudo teve como objetivo avaliar a prevalência da leishmaniose em cães de abrigo no município de Uberlândia, Minas Gerais. Foram selecionados 39 cães no abrigo da Associação Protetora de Animais (APA) e diagnosticados pelo teste triagem ALERE® Leishmania, sendo identificados dois animais soropositivos. Os mesmos foram também reagentes positivos ao teste confirmatório ELISA, sendo considerados doentes. A prevalência aparente observada foi de 5,12\% (IC 95\%: 1,42 - 16,89) e a identificação de casos confirmados da leishmaniose canina é útil para detectar áreas de riscos, e assim tomar medidas preventivas e de promoção em saúde.
\end{abstract}

PALAVRAS-CHAVE: Canis lupus familiaris, Leishmania, Protozoários, Saúde Pública.

\section{PREVALENCE OF CANINE LEISHMANIOSIS IN HOUSING DOGS IN THE MUNICIPALITY OF UBERLÍNDIA - MINAS GERAIS - CASE STUDY}

\begin{abstract}
Leishmaniasis is a major problem in global public health today and is an endemic disease in several regions of Brazil and the world, due to protozoa of the genus Leishmania, being the main species that cause visceral leishmaniasis in dogs and Leishmania infanti and L. chagasi, which are spread by the bite of the sandfly. The largest reservoirs of visceral leishmaniasis are domestic dogs. The increase in the number of dogs abandoned in the shelter has increased significantly, and the risk of spreading disease among these animals in the shelter has become constant. This
\end{abstract}


study aims to assess the prevalence of leishmaniasis in shelter dogs in the city of Uberlândia, Minas Gerais. 39 dogs were selected at the shelter of the Animal Protection Association (APA) and diagnosed by the ALERE® Leishmania screening test, and two seropositive animals were selected. They were also positive reagents to the ELISA confirmation test, is considered seropositive. The apparent prevalence observed was $5.12 \%(95 \% \mathrm{Cl}: 1,42-16,89)$ and the identification of cases confirmed by canine leishmaniasis is useful to detect risk areas and, therefore, to take preventive measures and promote health.

KEYWORDS: Canis lupus familiaris, Leishmania, Protozoa, Public Health.

\section{INTRODUÇÃO}

A Leishmaniose tornou-se um grande problema na saúde pública global e é uma doença de grande importância endêmica em várias regiões do Brasil e do mundo (FIGUEIREDO et al., 2017). Segundo Greene (2015), todo ano mais de dois milhões de casos novos de leishmaniose são registrados no mundo, segundo a Organização Mundial da Saúde, é uma doença infecciosa que acomete humanos e animais, tanto animais domésticos quanto selvagem no mundo todo. A região com maior prevalência é a região do nordeste, tendo o estado de Alagoas a maior prevalência e maior letalidade (REIS et al., 2017).

Essa patologia é causada por protozoários do gênero Leishmania, e as principais espécies que causam a leishmaniose visceral em cães são a Leishmania infantum e leishmaniose chagasi, e é disseminada pela picada do mosquito flebotomíneo da espécie Phetobotomus spp na Europa e Lutzomya longipalpis no Brasil, conhecido popularmente como mosquito palha (FERNANDES et al., 2016).

Os cães domésticos são os principais reservatórios da leishmaniose visceral que acomete os humanos, a infecção ocorre em áreas urbanas e rurais, o que representa uma ameaça à saúde animal e pública (LIMA et al., 2019). No Brasil, os mais altos números de casos são registrados nas regiões Norte e Nordeste pelo fato da precariedade sanitária da região, mas também está presente em várias cidades no Centro Oeste e Sudeste do país, causando epidemias urbanas e aumentando a letalidade (NERY et al., 2017). Assim pela incidência da doença no país, o risco de desenvolver a leishmaniose em uma população exposta é alta, isso implica na necessidade de implementação de medidas de controle de segurança (SANTOS et. al., 2017).

Animais infectados poderão desenvolver a doença dependendo da resposta imunológica, a qual determinará se esses animais desenvolverão uma infecção generalizada ou se a infecção irá progredir para um estado subclínico da doença. $A$ leishmaniose visceral canina possui sinais clínicos muito similares a de outras doenças, o que são desafios para o diagnóstico clínico, assim alguns métodos complementares são de grande valia para que o diagnóstico seja fechado (SANTOS et al., 2017).

O aumento do número de cães em situação de rua ou abandonados em abrigo tem aumentado bastante, e o risco de disseminação de doenças entre esses animais em abrigo tornou-se constante. O cão como principal reservatório da leishmaniose, gera uma situação preocupante, pois além da disseminação da doença para cães sadios apresenta risco de contaminação para a população humana. A importância do estudo de prevalência da leishmaniose, além de detectar a doença em abrigos, também é importante para controle de disseminação para animais sadios, futuros adotantes voluntários e vizinhança do abrigo que podem 
estar sujeitos a contaminação. Objetivou-se verificar a prevalência da Leishmaniose Canina em um abrigo de cães, situado no município de Uberlândia - Minas Gerais.

\section{MATERIAL E MÉTODOS}

O presente estudo teve os procedimentos avaliados e aprovados pelo Conselho de Ética no Uso de Animais/Unitri sob protocolo 47/2017-2. O estudo foi realizado na Associação de Proteção Animal (APA), situado no município de Uberlândia, Minas Gerais. Os animais abrigados pela APA, são animais que foram recolhidos por maus tratos, violência doméstica e sexual, filhotes abandonados e em risco de saúde. Atualmente na associação vivem aproximadamente 300 cães, entre machos e fêmeas, adultos e filhotes, que são alojados em baias coletivas onde os animais se alimentam exclusivamente de rações e água ad libitum.

Todos os animais que foram utilizados para a pesquisa são animais imunizados com a vacina Elevencell® que é composta por 11 antígenos (Cinomose, Parvovirose, Hepatite infecciosa, Adenovírus tipo 2 e Parainfluenza, Coronavírus e suspensões bacterianas inativadas de Leptospira interrogans sorotipo canicola, icterohaemorrhagiae, pomona, grippothyphosa e copenhageni) e antirrábica. Além da vacinação, o controle de ectoparasitas também é realizado com um manejo integrado ambiente e animal. Ocorre ainda o controle de endoparasitoses por meio de vermífugos na formulação comprimido.

Foram coletadas amostras de sangue por venopunção nas veias cefálicas ou safena, em 39 animais sem raça definida, sendo aleatório a coleta entre fêmeas e machos de animais com idade aproximadamente entre três e 10 anos, optando pelos animais mais dóceis com o objetivo de evitar métodos de contenções. Foram utilizadas seringas descartáveis de medida de $3 \mathrm{~mL}$ e agulha de calibre 25/7 e tubo para bioquímica sem anti-coagulante, que foram devidamente armazenados. Para extração do soro sanguíneo os tubos foram centrifugados e o soro armazenado em tubos de microaglutinação, em temperatura $-20{ }^{\circ} \mathrm{C}$ até execução dos testes.

Seguiu-se o seguinte roteiro de diagnóstico: teste rápido de triagem ALERE®, e os casos positivos encaminhados para execução do teste confirmatório pelo ELISA, sendo considerado doente 0 animal que for reagente positivo no teste confirmatório.

As amostras foram submetidas ao teste rápido Alere ${ }^{\circledR}$ Leishmania Ac Test Kit é um imunoensaio imunocromatográfico teste para detecção qualitativa de anticorpos anti leishmania infantum, através de antígenos rK9, rK39 e rK36 em amostras de soro, plasma ou sangue total canino, com resultados positivo ou negativo, para diagnóstico do animal. O teste oferece o resultado em cerca de 20 minutos e dispensa estrutura laboratorial e equipamentos (MARCELINO; SOUZAFILHO, 2015), sendo executado no Laboratório de Análises Clínicas do Centro Universitário do Triângulo. Para os animais positivos com o teste rápido, foi empregado o teste de diagnóstico confirmatório ELISA, realizado pelo laboratório TECSA.

A base de dados, assim como a construção de tabelas e gráficos foi realizada através das planilhas eletrônicas do programa Microsoft Excel versão 2013. Na base de dados, cada amostra de soro sanguíneo possui o município, data de coleta, idade, raça, sexo, espécie, resultados do teste de triagem e confirmatório.

Prevalência de animais infectados: A prevalência aparente $(\mathrm{Pa})$ e prevalência real (Pr) para animais foram calculadas conforme Lemos et al. (2015). A $\mathrm{Pa}$ foi obtida ponderando-se a frequência com o peso que cada propriedade tem em número de animais em relação ao total de propriedades (fator de ponderação). Os 
valores de Pr foram estimados ajustando-se o valor da $\mathrm{Pa}$ para a especificidade (100\%) e sensibilidade (98,0\%) do teste de ELISA (LEMOS et al., 2015).

$\operatorname{Pr}=(\mathbf{P a}+$ Esp-1) $/($ Sen + Esp-1), onde:

Sen = sensibilidade do teste;

$\mathrm{Pa}=$ prevalência aparente;

Esp = especificidade do teste.

Os intervalos de confiança (IC) das prevalências aparente e real de animais e de rebanhos foram calculados para uma confiança de 95\%, conforme Martin et al. (1987), por meio da seguinte fórmula:

IC = valor estatístico $\pm(z$ * EP), onde:

$z=1,96$ (SAMPAIO 2007);

$\mathrm{EP}=$ erro-padrão

\section{RESULTADOS E DISCUSSÃO}

Dos 39 cães que foram amostrados, diagnosticou-se como soropositivos pelo teste triagem ALERE® dois animais e em seguida foram também reagentes positivos ao teste confirmatório ELISA e os valores de prevalência são apresentados na tabela 1.

TABELA 1: Prevalência aparente, intervalo de confiança, prevalência real de Leishmaniose visceral canina de um abrigo coletivo, Uberlândia, MG, 2018.

\begin{tabular}{lrrl}
\hline $\mathrm{Pa}$ & $\mathrm{IC}$ & $\mathrm{IC}$ & $\mathrm{Pr}$ \\
& inferior $\mathrm{Pa}$ & superior $\mathrm{Pa}$ & \\
\hline $5,12 \%$ & $1,42 \%$ & $16,89 \%$ & $5,23 \%$ \\
\hline
\end{tabular}

Segundo Maywald et al. (1996) que estudou a prevalência de leishmaniose no município de Uberlândia, no exame clínico não detectou cães domésticos com sinais clínicos evidentes. Quanto à pesquisa de anticorpos circulantes no Município de Uberlândia, a RIFI detectou $11(4,8 \%)$ cães positivos para a pesquisa de anticorpos anti-Leishmania, num total de 230 testados. Ainda segundo estes mesmos autores em área urbana do Município, nove cães (4,5\%) foram soropositivos em um total de 200 soros testados. Na zona rural, dos 30 cães examinados, dois $(6,6 \%)$ apresentaram-se reagentes na RIFI.

Estudo mais recente demonstrou uma prevalência em área urbana de 0,66\% em um total de 7236 animais avaliados pelo Centro de Controle de Zoonoses (CCZ) da cidade de Uberlândia, tendo casos detectados na zona oeste onde está localizada a APA (CORTES et al., 2020). O roteiro de diagnóstico empregado pelo CCZ foi o mesmo adotado no presente trabalho.

Alguns ambientes que apresentam características ambientais e socioculturais que propiciam ambientes favoráveis para a manutenção do mosquito como ambientes em moradias próximos de matas (SILVA et al., 2016; SILVA et al., 2017), o que pode explicar os animais soropositivos da região de Uberlândia (MG), pois os animais assistidos no abrigo são animais errantes, provenientes de abandonos em área rural e urbana, onde previamente o animal entrou em contato com o vetor flebotomíneo. 
De acordo com Monteiro et al. (2005), as transformações ambientais como urbanização e êxodo rural, acarretam a expansão e o aparecimento de novos focos e facilitada a ocorrência de epidemias. Na cidade de Uberlândia não tem sido diferente, com a expansão econômica vivida nos últimos anos, aproximando a urbanização das áreas de ocorrência do vetor. Em estudos conduzidos ao longo da década de 2000 foi possível observar a urbanização e domiciliação do principal vetor, Lutzomia longipalpis (LEMOS et al., 2004; PAULA et al., 2008), fator esse é potencial impacto à saúde humana visto que há animais doentes e o vetor presente.

O manejo de resíduos orgânicos com adequada destinação associado ao controle químico com inseticidas são as principais estratégias para o controle da transmissão da leishmaniose visceral (BRITO et al., 2016). A eutanásia de animais positivos realizada de modo isolado, sem controle do vetor, não demonstra resultado sobre o controle da doença.

Por serem animais assistidos por médicos veterinários, ambiente ausente da presença do vetor optou-se pelo tratamento dos animais com miltefosina, uso de coleira repelente e o monitoramento parasitológico para demonstrar a erradicação do parasita no animal.

\section{CONCLUSÕES}

Pode-se concluir que a presença de animais positivos neste abrigo corrobora com a prevalência da doença na cidade de Uberlândia e sua expansão territorial para áreas afastadas do primeiro caso autóctone detectado em 2008 na zona leste do município, visto que o abrigo se encontra na zona oeste do município. Novas estratégias de controle precisam ser implementadas pelos serviços de saúde com integração entre as áreas de vigilância e ações com uma abordagem em Saúde Única.

\section{REFERÊNCIAS}

BRITO F.G.; LANGONI, H.; SILVA, R. C.; ROTONDANO, T. E. F.; MELO, M. A. et al. Canine visceral leishmaniasis in the Northeast Region of Brazil. Journal of Venomous Animals and Toxins including Tropical Diases factor. V: 22, 15. 2016. Disponivel em: http://dx.doi.org/10.1186/s40409-016-0069-4

CORTES, L. F. M., SOUSA, D. M. A.; JUNQUEIRA JUNIOR, D. G.; BORGES, G. L. F. N.; TEIXEIRA, P. A. Prevalence of visceral leishmaniasis in dogs in Uberlandia, Minas Gerais State, Brazil, 2020 [in press]

FERNANDES A. R. F.; PIMENTA, C. L. R. M.; VIDAL, I. F.; OLIVEIRA, G. C.; SARTORI, R. S. et al. Risk factors associated with seropositivity for Leishmania spp. and Trypanosoma cruzi in dogs in the state of Paraiba, Brazil. Brazil Journal

$\begin{array}{llllll}\text { Veterinary Parasitology. } & \text { V. } & 25 & \text { p. } & 90-98 . & 2016\end{array}$ Disponível em: http://www.scielo.br/scielo.php?script=sci_arttext\&pid=S198429612016000100090 DOI: 10.1590/S1984-29612016010

FIGUEIREDO, A. B. F.; WERNECK, G. L.; PIRES E CRUZ, M. S.; SILVA, J. P.; ALMEIDA, A. S. Uso e cobertura do solo e prevalência de leishmaniose visceral canina em Teresina, Piauí, Brasil: uma abordagem utilizando sensoriamento remoto orbital. Caderno Saúde Pública, v..33, n.10, Rio de Janeiro 2017, 2017. Dispomível em: http://www.scielo.br/scielo.php?pid=S0102311X2017001005010\&script=sci_abstract\&tlng=pt. DOI: $\quad$ /10.1590/0102$311 \times 00093516$ 
GREENE, C E. Doenças Infecciosas em Cães e Gatos. 4ํE. SP Roca, 2015.

LEMOS, E. M.; LAURENTI, M. D.; MOREIRA, M. A.; REIS, A. B.; GIUNCHETTI, R. C. et al. Canine visceral leishmaniasis: performance of a rapid diagnostic test (Kalazar Detect) in dogs with and without signs of the disease. Acta Tropical. 2015

LEMOS, J. C., LIMA, S.C., NETO, A.A.P, CASAGRANDE, B., VIEIRA, G.S.S. et al. Encontro de Lutzomyia longipalpis na área de implantação da usina hidrelétrica Capim Branco I, na bacia do rio Araguari, no município de Uberlândia, Minas Gerais - Brasil. Revista Caminhos de Geografia, v.12, p.186-198, 2004. Disponível em: http://www.seer.ufu.br/index.php/caminhosdegeografia/article/view/15336

LIMA, D.A., NOVO, S.P.C., SANTOS, F.N., MACIEL, E.M.S.G. Aspectos epidemiológicos, sociais e ambientais relacionados a transmissão e ao controle da leishmaniose visceral canina na Ilha de Marambaia, Mangaratiba-Rio de Janeiro. Revista Saúde e Meio Ambiente - RESMA, Três Lagoas, v. 9, n. 3, p. 64-81, Agosto Dezembro. 2019.

MARCELINO, A. P.; SOUZA-FILHO, J. A., Instruções para a realização do teste rápido imunocromatográfico Alere para diagnostico sorológico da leishmaniose visceral canina. Belo Horizonte,2015. disponivel em : HTTP://WWW.FUNED.MG.GOV.BR/WPCONTENT/UPLOADS/2015/09/INSTRU\%C 3\%A7\%C3\%B5ES-TESTE-RAPIDO-ALERE-LVC-MANUAL.PDF. Acesso em: $11 / 06 / 2018$

MARTIN, S. W.; MEEK, A. H.; WILLEBERG, P. Veterinary Epidemiology; principles and methods. Ames: lowa State University,. p. 343 - 1987 Disponivel em: https://vtechworks.lib.vt.edu/bitstream/handle/10919/72274/Vet_Epidem_Full_Textbo ok.pdf? sequence $=4 \&$ isAllowed $=y$

MAYWALD, P. G.; MACHADO, M. I. COSTA-CRUZ, J. M.; GONÇALVES-PIRES, M. R. F. Leishmaniose tegumentar, visceral e doença de Chagas caninas em municípios do Triângulo Mineiro e Alto Paranaíba, Minas Gerais, Brasil. Caderno de Saúde Pública, Rio de Janeiro, 12(3) p. 321-328, jul-set, 1996 Disponível em: http://www.scielo.br/scielo.php?script=sci_abstract\&pid=S0102$311 \times 1996000300005 \& \operatorname{lng}=e n \& n r m=i s o \& t \operatorname{lng}=p t \quad$ DOI: $\quad 10.1590 / S 0102-$ $311 \times 1996000300005$.

MONTEIRO, E. M.; SILVA, J. C. F.; COSTA, R. .T; COSTA, D. C.; BARATA, R. A. et al. Leishmaniose visceral: estudo de flebotomíneos e infecção canina em Montes Claros, Minas Gerais Visceral leishmaniasis. Revista da Sociedade Brasileira de Medicina Tropical V. 38(2) p. 147-152, mar-abr, 2005 Disponível em: http://www.scielo.br/scielo.php?script=sci_arttext\&pid=S003786822005000200004 DOI:10.1590/S0037-86822005000200004

NERY, G., BECERRA, D. R. D.; BORJA, L. S.; MAGALHAES-JUNIOR, J. T.; SOUZA, B. M. P. S. et al. Avaliação da infectividade parasitária a Lutzomyia longipalpis por xenodiagnóstico em cães tratados para leishmaniose visceral naturalmente adquirida. Pesquisa Veterinária Brasileira. V. 37 N.7 Rio de Janeiro July 2017.

Disponível em: http:/www.scielo.br/scielo.php?pid=S0100- 
736×2017000700009.

PAULA, M. B. C.; RODRIGUES, E. A. S.; SOUZA, A. A.; REIS, A. A.; PAULA, F. P. et al. Primeiro encontro de Lutzomyia longipalpis (Lutz \& Neiva, 1912) na área urbana de Uberlândia, MG, concomitante com o relato de primeiro caso autóctone de leishmaniose visceral humana. Revista da Sociedade Brasileira de Medicina Tropical vol. 41, n. 3, pp. 304-305, mai-jun, 2008. Disponível em: http://www.scielo.br/scielo.php?pid=S0037-

86822008000300016\&script=sci_abstract\&tIng=pt

REIS, L. L.; BALIEIRO, A. A. da S.; FONSECA, F. R.; GONÇALVES, M. J. F. Alterações na epidemiologia da leishmaniose visceral no Brasil no período de 2001 a 2014. Revista da Sociedade Brasileira de Medicina Tropical. Vol. 50, n. 5, pp. 638-645.

Disponível em: http://www.scielo.br/scielo.php?script=sci arttext\&pid=S003786822017000500638 DOI: 10.1590/0037-8682-0243-2017

SAMPAIO, M.B.I., Estatística Aplicada à Experimentação Animal. Vol.1. $3^{\text {a }}$ ed. FEP-MVZ, Belo Horizonte, p. 264. 2007

SANTOS, H. D., GALVÃO, S.R., DIAS, F.E.F., RIBEIRO, T.M.P, NEGREIROSFILHO, O., et al. Alta frequência de leishmaniose visceral em cães sob cuidados clínicos veterinários em uma área de transmissão intensa no estado de Tocantins, Brasil. Ciência Rural V. 47 N.3 ,05 de janeiro de 2017. Diponível em: http://www.scielo.br/scielo.php?pid=S010384782017000300501\&script=sci_abstract\&tlng=pt DOI: 10.1590/0103$8478 \mathrm{cr} 20160260$.

SILVA R. B. S., MENDES, R. S.; SANTANA, V. L.; SOUZA, H. C.; RAMOS, C. P. S. et al. Aspectos epidemiológicos da leishmaniose visceral canina na zona rural do semiárido paraibano e análise de técnicas de diagnóstico. Pesquisa Veterinária

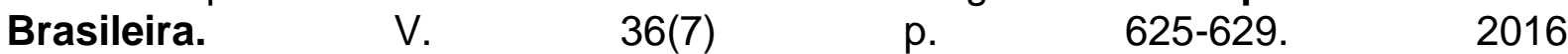
Disponível em: http://www.scielo.br/scielo.php?pid=S0100736X2016000700625\&script=sci_abstract\&tlng=pt DOI: $10.1590 /$ S0100736X2016000700011.

SILVA, J. D.; MELO, D. H. M.; COSTA, J. A. G.; COSTA, D .F.; SILVA, R. B. S. et al. Leishmaniose visceral em cães de assentamentos rurais. Pesquisa Veterinária Brasileira. v.37 no.11 Rio de Janeiro Nov. 2017. Disponível em: http://www.scielo.br/scielo.php?script=sci_abstract\&pid=S0100736X2017001101292\&lng=en\&nrm=iso\&tlng=pt DOI: $\quad 10.1590 /$ s0100$736 \times 2017001100016$. 